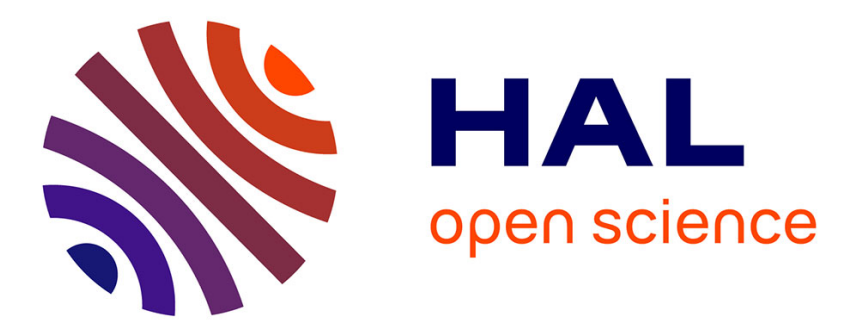

\title{
Grain boundary premelting in crystalline benzene as studied by proton N.M.R.
}

\author{
C.J. Craven, G. Dosseh, B. Rousseau, Alain H. Fuchs
}

\section{To cite this version:}

C.J. Craven, G. Dosseh, B. Rousseau, Alain H. Fuchs. Grain boundary premelting in crystalline benzene as studied by proton N.M.R.. Journal de Physique, 1990, 51 (21), pp.2489-2499. 10.1051/jphys:0199000510210248900 . jpa-00212546

\section{HAL Id: jpa-00212546 https://hal.science/jpa-00212546}

Submitted on 1 Jan 1990

HAL is a multi-disciplinary open access archive for the deposit and dissemination of scientific research documents, whether they are published or not. The documents may come from teaching and research institutions in France or abroad, or from public or private research centers.
L'archive ouverte pluridisciplinaire HAL, est destinée au dépôt et à la diffusion de documents scientifiques de niveau recherche, publiés ou non, émanant des établissements d'enseignement et de recherche français ou étrangers, des laboratoires publics ou privés. 
Classification

Physics Abstracts

$76.60 \mathrm{E}-61.65$

\title{
Grain boundary premelting in crystalline benzene as studied by proton N.M.R.
}

\author{
C. J. Craven (*), G. Dosseh, B. Rousseau and A. H. Fuchs (**) \\ Laboratoire de Chimie Physique des Matériaux Amorphes (***), Bâtiment 490, Université Paris- \\ Sud, F-91405 Orsay, France
}

(Received 17 January 1990, revised 6 July 1990, accepted 18 July 1990)

\begin{abstract}
Résumé. - Le phénomène de préfusion dans le benzène polycristallin de pureté variable a été étudié par r.m.n. du proton. Nos résultats suggèrent que la préfusion débute aux joints de grains. La nature exacte des domaines fondus n'est pas encore très claire mais il semble que l'on observe un liquide d'interface très corrélé, comme les auteurs qui étudient la fusion de surface.
\end{abstract}

\begin{abstract}
The phenomenon of premelting has been investigated by proton n.m.r. in polycrystalline benzene of various purity. Our results suggest that premelting initiates at grain boundaries. The exact nature of the molten domains near the standard melting point is not yet clear but is presumably analogous to what is obtained in the surface melting experiments, i.e. a «strongly correlated interface liquid ».
\end{abstract}

\section{Introduction.}

It is now recognized that melting can initiate at crystalline imperfections. Surface premelting has been observed in pure single crystals of metals [1] as well as in other model systems such as for instance thin films of methane adsorbed on graphite or $\mathrm{MgO}[2,3]$. Whether this effect dominates other premelting effects (such as the initiation of melting around impurities) in ordinary polycristalline materials is still an open question. Recently [4], a proton absorption line study of crystalline thiophene $\left(\mathrm{C}_{5} \mathrm{H}_{4} \mathrm{~S}\right)$ has shown that, a few kelvin below the nominal bulk melting point, $T_{\mathrm{m}}$, a composite spectrum is observed consisting of a narrow line superimposed on a broad line of $\sim 10 \mathrm{kHz}$ width corresponding to the orientationally disordered phase. The intensity of this narrow line rises with increasing temperature at the expense of the broad component. At $T_{m}$, the ordinary high resolution liquid spectrum is observed. We suggested that the narrow component could be assigned to local mobility domains lying near crystal defects, but we were not able to establish which among the various kind of lattice imperfections (point defects, dislocations, grain boundaries, free surface) were mainly responsible for the observed feature.

(*) Permanent address : Department of Physics, University of Edinburgh, King's Building, Edinburgh, EH9 3JZ, Scotland.

$(* *)$ Author to whom correspondence should be addressed.

$(* * *)$ URA 1104 CNRS. 
In this work, the nature of the mobility domains involved in the observed narrow line near $T_{\mathrm{m}}$ is investigated in the case of polycrystalline benzene in which the molecular dynamics feature is essentially analogous to that of thiophene. The study of benzene-heptane mixtures, in which heptane acts as a solid-insoluble impurity shows that melting is likely to initiate at grain boundaries. The observed narrow line is assigned to a "pseudo-liquid " lying at the interface between grains. It is also pointed out that very small amounts of solid-insoluble impurities $(\leqslant 200 \mathrm{ppm})$ contribute to the extent of the grain boundary premelting. As far as thiophene and benzene are concerned, an "internal interface » premelting model seems to hold. The "surface melting " findings for single crystals could then be extended, at least in these cases, to polycrystalline samples.

\section{Experimental.}

A commercially available Fourier transform NMR spectrometer, type Bruker AM400, operating at $400.13 \mathrm{MHz}$ (proton resonance frequency), was used in this work. Short frequency pulses of $2 \mu \mathrm{s}$ were used in order to avoid signal distorsion due to power inhomogeneity (the $\mathrm{H}_{1}$ intensity is 11.9 Gauss). 16 F.I.D. were accumulated at each temperature giving rise to a $\mathrm{S} / \mathrm{N}$ ratio of roughly 40 . The overall recovery time of the probe and receiver was $5 \mu$ s so that no more than about $10 \%$ of the signal (obtained with quadrature detection) was lost. The spin-spin relaxation times $T_{2}$ have been obtained through the usual $(90, \tau, 180)$ Hahn technique. The $T_{1}$ have been obtained using the inversion-recovery method.

Cryostatic temperatures in the apparatus are obtained by passing evaporated liquid nitrogen past the sample tube. In order to measure the sample temperature, a CopperConstantan thermocouple was positioned next to the sample tube and use was made of an empirically derived chemical shift formula for liquid Methanol [5] enabling temperature calibration to an accuracy of better than $+/-0.5 \mathrm{~K}$. Although when using a gas flow, a temperature gradient must exist across the sample region, we have found that at relatively high values of the nitrogen flow rate, the temperature difference across the sample is better than $0.1 \mathrm{~K}$. This was checked by placing thermocouples at both ends of the sample inside the tube. No contact can occur between the spinning sample and the r.f. coil thus avoiding local thermal perturbation. Temperature stability in the sample is also known to be better than $+/-0.05 \mathrm{~K}$.

All the spectra shown below have been recorded on heating after a rapid cooling $\left(\sim 10 \mathrm{~K} \mathrm{~min}^{-1}\right)$ of the liquid sample. When cooled rapidly enough, benzene is known to form a perfect polycristalline sample [8]. The effect observed when changing the heat treatment is described below in the discussion section.

Benzene was purchased from Merck. Samples of different purities ( 99.6 to $99.98 \%$ ), degased and sealed under vacuum, have been studied, as well as samples containing heptane as the main solid-insoluble impurity ( 0.1 to $6 \%$ heptane). Heptane was chosen because the benzene-heptane phase diagram is known [6]. Heptane is totally soluble in liquid benzene and totally insoluble in the solid phase. The coordinates of the eutectic point at atmospheric pressure is :

$$
T=182 \mathrm{~K}, x \text { (benzene) }=0.03
$$

\section{Results.}

The evolution of the proton lineshape near melting is shown in figure 1 for a sample of $99.98 \%$ purity. The narrow line appearing on top of the broad component has a width of $\sim 500 \mathrm{~Hz}$, much larger than that of the supercooled liquid in the same temperature range 


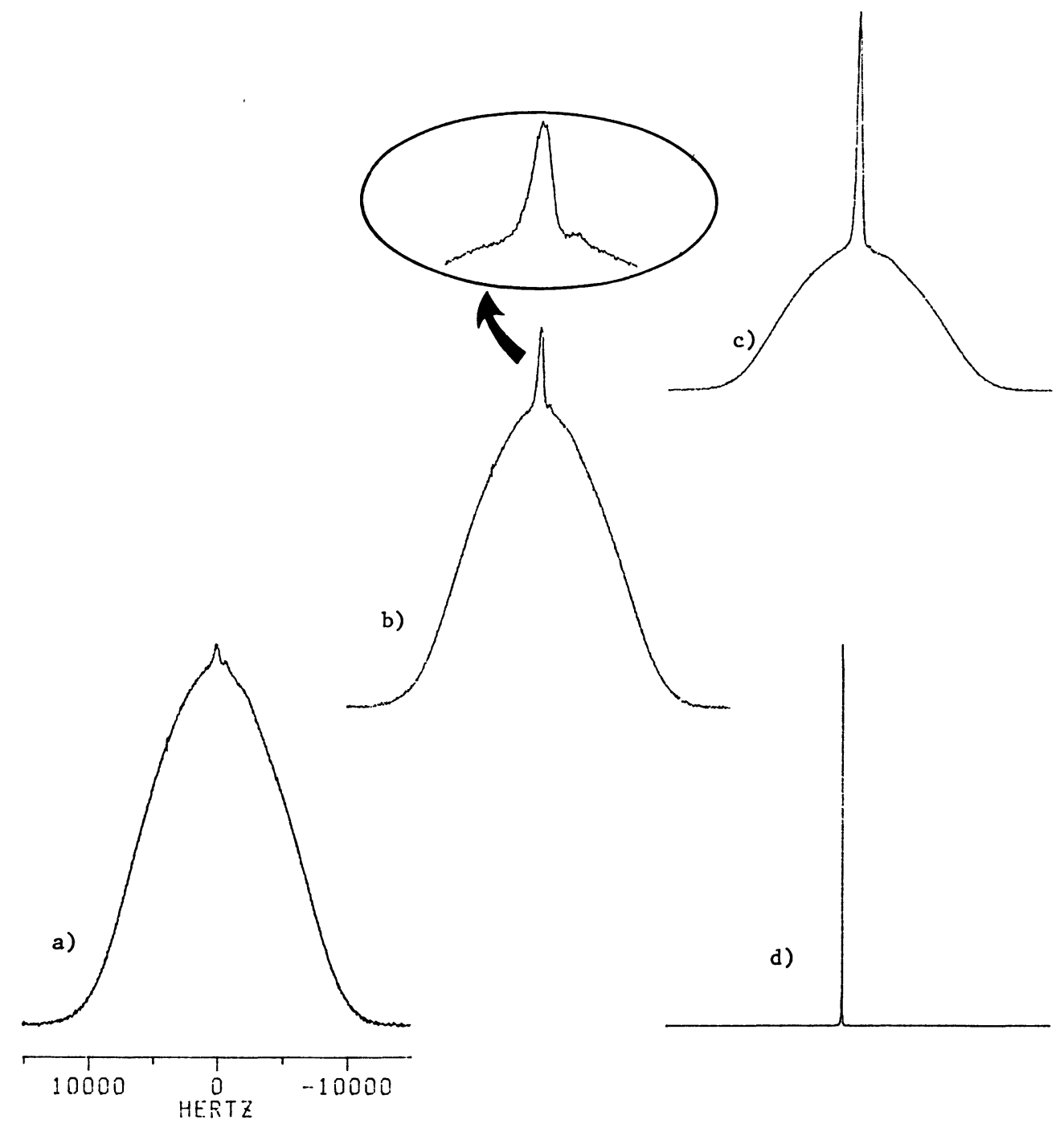

Fig. 1. - Evolution of the proton n.m.r. lineshape near melting in a sample of $99.98 \%$ polycristalline benzene. a) $T_{\mathrm{m}}-5 \mathrm{~K}$, b) $T_{\mathrm{m}}-1 \mathrm{~K}$. The narrow line is shown above (enlargement). The little peak just beside the narrow line is an artefact, it appears with the same intensity in absence of protonated sample. c) $T_{\mathrm{m}}-0.1 \mathrm{~K}$, d) supercooled liquid, $T_{\mathrm{m}}-3 \mathrm{~K} . T_{\mathrm{m}}$ is the standard melting point.

$(<5 \mathrm{~Hz})$. The width of the narrow line remains constant throughout the premelting region. As in the case of thiophene [4], this line can only be assigned to molecular self-diffusion since isotropic molecular tumbling alone would lead to a width of not less than $5 \mathrm{kHz}$ [7]. On the other hand the broad line of $\sim 10 \mathrm{kHz}$ can easily be understood in terms of fast in-plane molecular reorientation. The unexpected feature lies in the occurrence of a composite spectrum near melting reflecting the existence long-lived mobility domains in the crystal.

A sample of $99.6 \%$ purity has also been studied. The same qualitative behaviour was found. However the proportion of the molecules involved in the narrow line is a slightly (but significantly) larger in this sample than in the purest one, whatever the temperature. This is shown in figure 2. An impurity effect on melting is usually attributed to solid-insoluble 


\section{$\%$ narrow line}

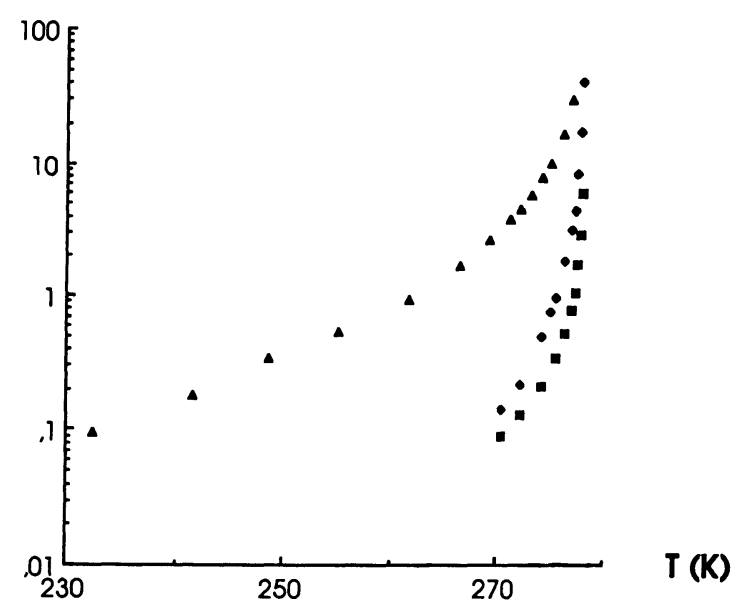

Fig. 2. - Proportion of the molecules involved in the $\sim 500 \mathrm{~Hz}$ narrow line as a function of temperature for samples of different purities : $(\square) 99.98 \%,(\bullet) 99.6 \%$, ( $\Delta)$ benzene with $x$ (heptane $)=0.002$. The data are obtained through a graphical deconvolution of the composite lines shown in figures 1 and 3.

impurities (the so-called Raoult effect), this is why we have decided to study samples containing known amounts of heptane. Figure 3 shows the lineshape obtained for a sample with $x$ (heptane) $=0.05$, at $267 \mathrm{~K}$. Two narrow components corresponding to fast selfdiffusing benzene and heptane can be observed. The intensity of the heptane line remains constant with temperature indicating that all the heptane is involved in this narrow line. The corresponding line-width are very similar so is their temperature dependence, shown in figure 4. Unlike the heptane-free samples, the line-widths do not remain constant through the premelting range. At a temperature $T^{*}$, the widths begins to decrease and its value, above $T^{*}$, tends towards that of an ordinary liquid. The value of $T^{*}$ depends on the heptane concentration, as shown in figure 5. We have found that, at $T^{*}$, the total proportion of the self-diffusing molecules in the sample responsible for both the benzene and heptane narrow lines is equal to $(4+/-0.5) \%$, whatever the mole fraction of heptane, from 0.001 to 0.06 .

A Hahn spin-echo can be obtained from both the benzene and the heptane narrow components. Spin-spin relaxation times $T_{2}$ have been measured as a function of temperature in samples containing different proportions of heptane from 0.1 to $6 \%$. We have also measured $\mathrm{T}_{2}$ for the narrow heptane line in a sample containing $1.5 \%$ of heptane in deuterated benzene. Spin-spin relaxation times were found to be essentially identical for benzene and heptane. The temperature dependence of $T_{2}$ follows that of $T_{2}^{*}=(1 / \pi w)$, where $w$ is the full width at half height, as shown in figure 6. Below $T^{*}$, we have always found a proton $T_{2}$ value $(\sim 7 \mathrm{~ms})$ roughly 7 times larger than $T_{2}^{*}$. This is discussed in the following section, it can be explained in terms of an internal demagnetization field effect.

\section{Discussion.}

The nature of the mobility domains observed near melting, both in presence and in absence of heptane, is the main question raised by the present work.

The heptane-benzene phase diagram [6] can be used to solve this problem when a small proportion of heptane is present in the sample. Figure 7 shows the proportion of the mobile 


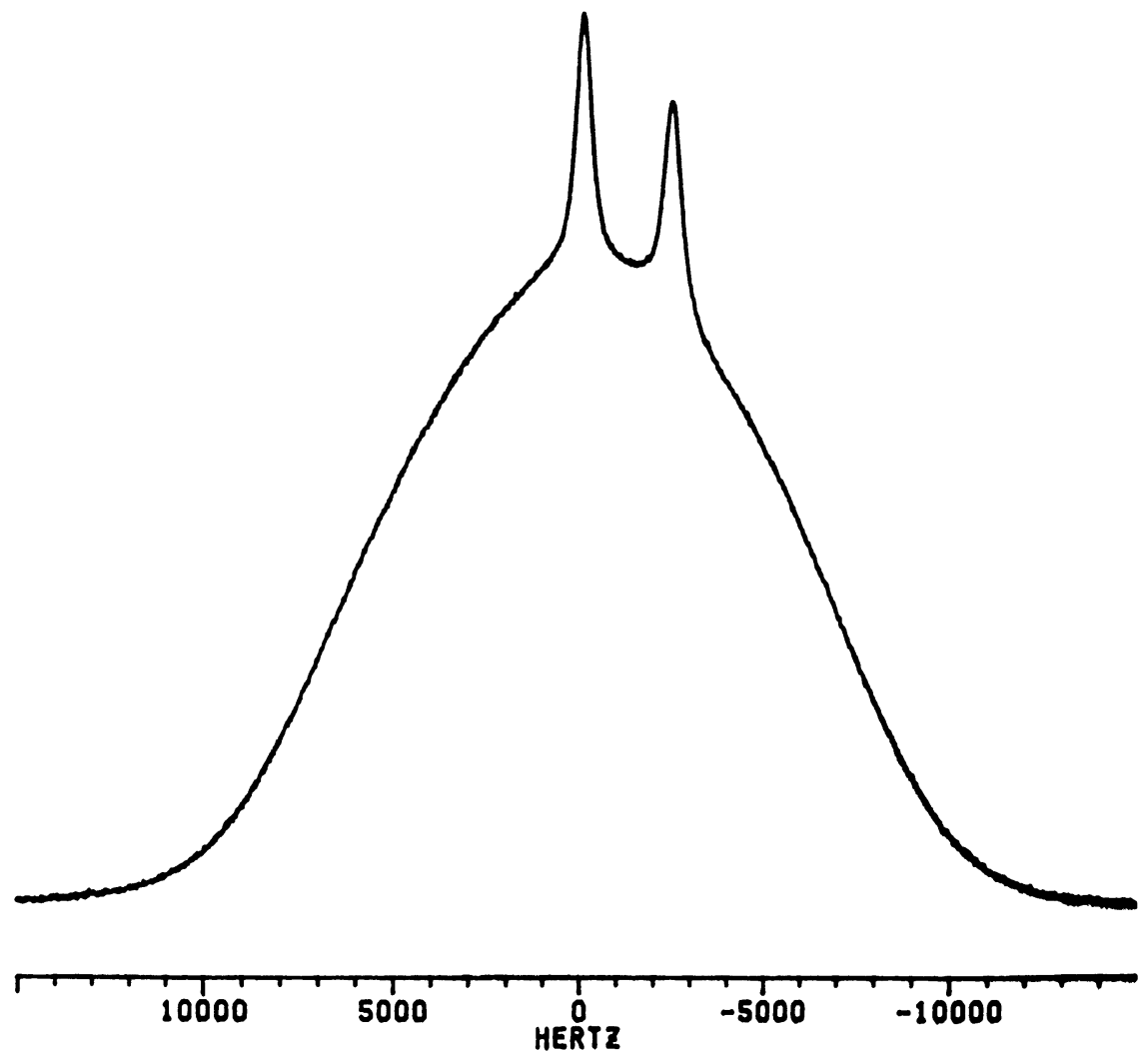

Fig. 3. - Proton absorption line of an heptane-benzene mixture with $x$ (heptane) $=0.05$ at $267 \mathrm{~K}$.

\section{Line width $(\mathbf{H z})$}

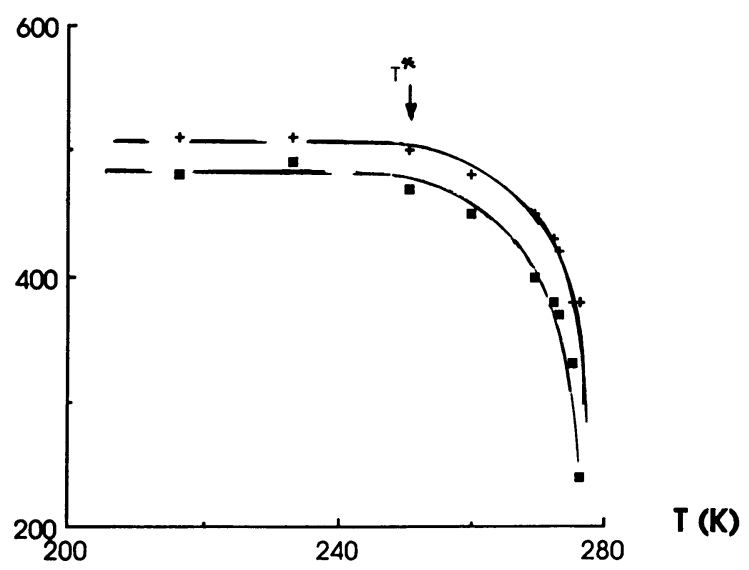

Fig. 4. - Temperature dependence of the linewidth of the narrow lines of benzene ( $\square)$ and heptane (+) in a sample with $x$ (heptane $)=0.05$. 


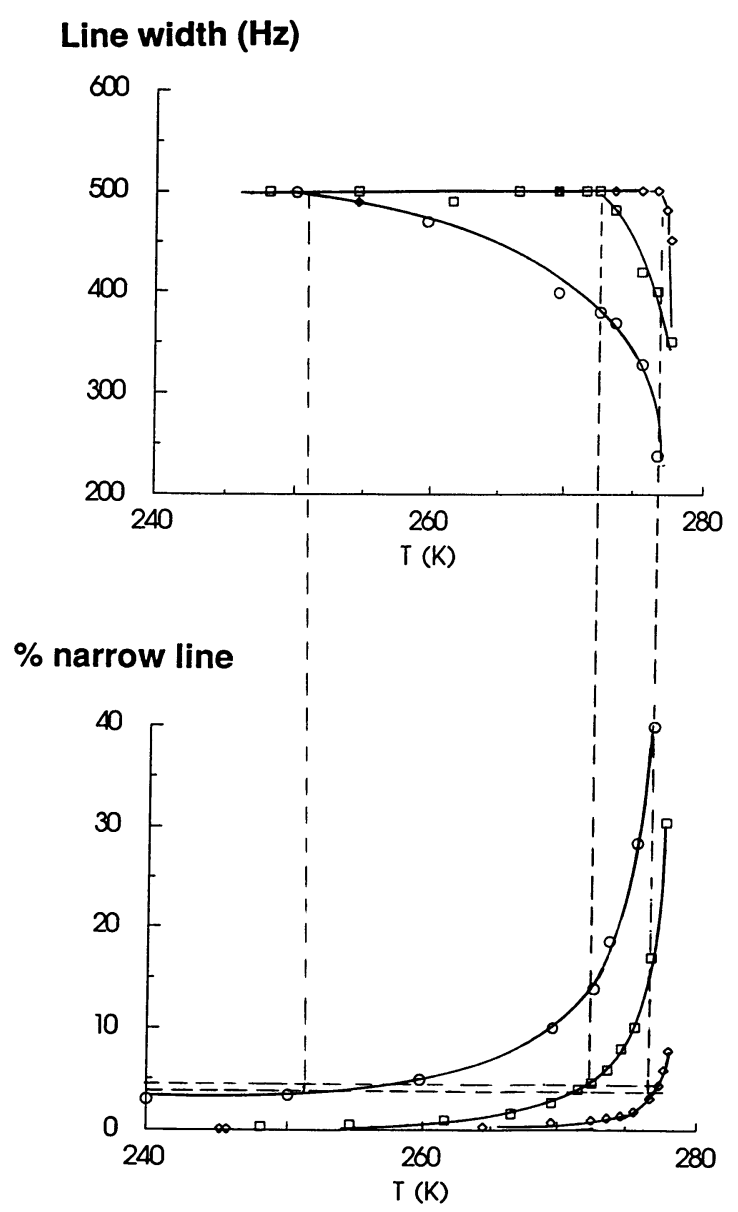

Fig. 5. - Temperature dependence of the proton n.m.r. linewidth of the benzene narrow component and proportion of the narrow lines for samples of different composition: $(\diamond) x$ (heptane $)=$ 0.002 , ( $\square$ ) $x$ (heptane $)=0.005$, (O) $x$ (heptane ) $=0.01$. The temperature (named $T^{*}$ in the text) at which the narrow component's width begins to decrease corresponds to a proportion of mobile molecules of $4+/-0.5 \%$.

molecules involved in the narrow lines as a function of temperature together with the proportion of liquid phase calculated from the phase diagram, using the so-called «lever rule ", in a sample containing $0.2 \%$ of heptane. The coincidence of both sets of data shows that the mobile domains are actually made of what is defined as a «liquid » in the differential scanning calorimetry experiment.

However, the observed spin-spin relaxation data shown in figure 6 do not fit with what is expected from an ordinary liquid. The constant $T_{2}$ value below $T^{*}$ clearly demonstrates that the direct dipole-dipole intermolecular interactions are not fully averaged by the molecular motions. This is confirmed by the proton $\left(T_{1}, T_{2}\right)$ data shown in figure 8 for heptane in the sample containing $1.5 \%$ of heptane in deuterated benzene. Such a feature is typical of a crystal with fast self-diffusion or of an adsorbed or viscous liquid.

In summary, the molecular mobility domains have both solid-like and liquid-like behaviour. This apparent contradiction can be solved by considering that the solid-insoluble 


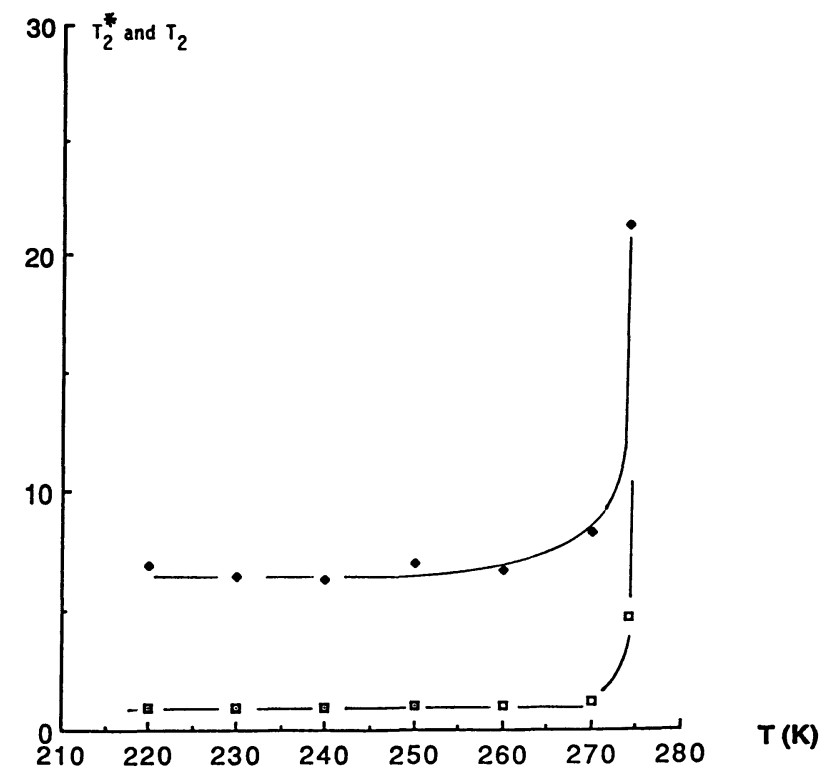

Fig. 6. - Spin-spin relaxation time $T_{2}(\diamond)$ and $T_{2}^{*}=(1 / \pi w)(\square)$, where $w$ is the full width at half height, as a function of temperature for the benzene narrow line in a sample with $x$ (heptane) $=$ 0.005 .

solute (i.e. heptane in our case), stands at the grain boundaries. It is then likely that the molten domains grow at the interface between grains. The fact that the proportion of the mobile molecules is the same at $T^{*}$, whatever the composition of the sample, suggests a simple picture in which a quasi-liquid film appears around the crystallites. The thickness of the film increases with temperature and there is a critical thickness, corresponding to a mean critical number of molecular layers $N^{*}$, above which the mobility progressively tends towards the diffusivity of the bulk liquid. In this tentative model, a straightforward calculation, assuming a uniform liquid film around the crystallites, shows that the mean grain diameter $D$ is related to $N^{*}$ through the relation $N^{*}=(D / 250)$, where $D$ is in Angstroms. The actual size of the crystallites in our experiments is not known. However a mean grain diameter of 500 to $1000 \mathrm{~A}$ is not unusual. This corresponds to two to four molecular layers at $T^{*}$. The above picture is analogous to what is described in the surface melting experiments of Frenken [1], Bienfait [2,3] and coworkers. The general idea which is supported by these results is that, in many ways (for instance transport properties), grain boundaries are analogous to surfaces.

We have tried to test this model by changing the grain size using different heat treatments. Carefully annealed samples systematically showed lower proportions of the mobile phase at the annealing temperature (a few kelvin below $T_{\mathrm{m}}$ ), in agreement with what we expected. We failed however to obtain quantitatively reproducible results, due to the unsuitability of our experimental device for this kind of experiments. Experiments with single crystals are planned using another spectrometer.

The extra-broadening of the narrow line observed below $T^{*}$, which leads to the difference of roughly an order of magnitude between $T_{2}$ and $T_{2}^{*}$, can be explained by the fact that, according to our picture, the mobile phase would gradually form a three-dimensional network of narrow liquid-like domains extending across the whole sample and give rise to an inhomogeneity of the internal demagnetization field. According to Drain [12], a field 


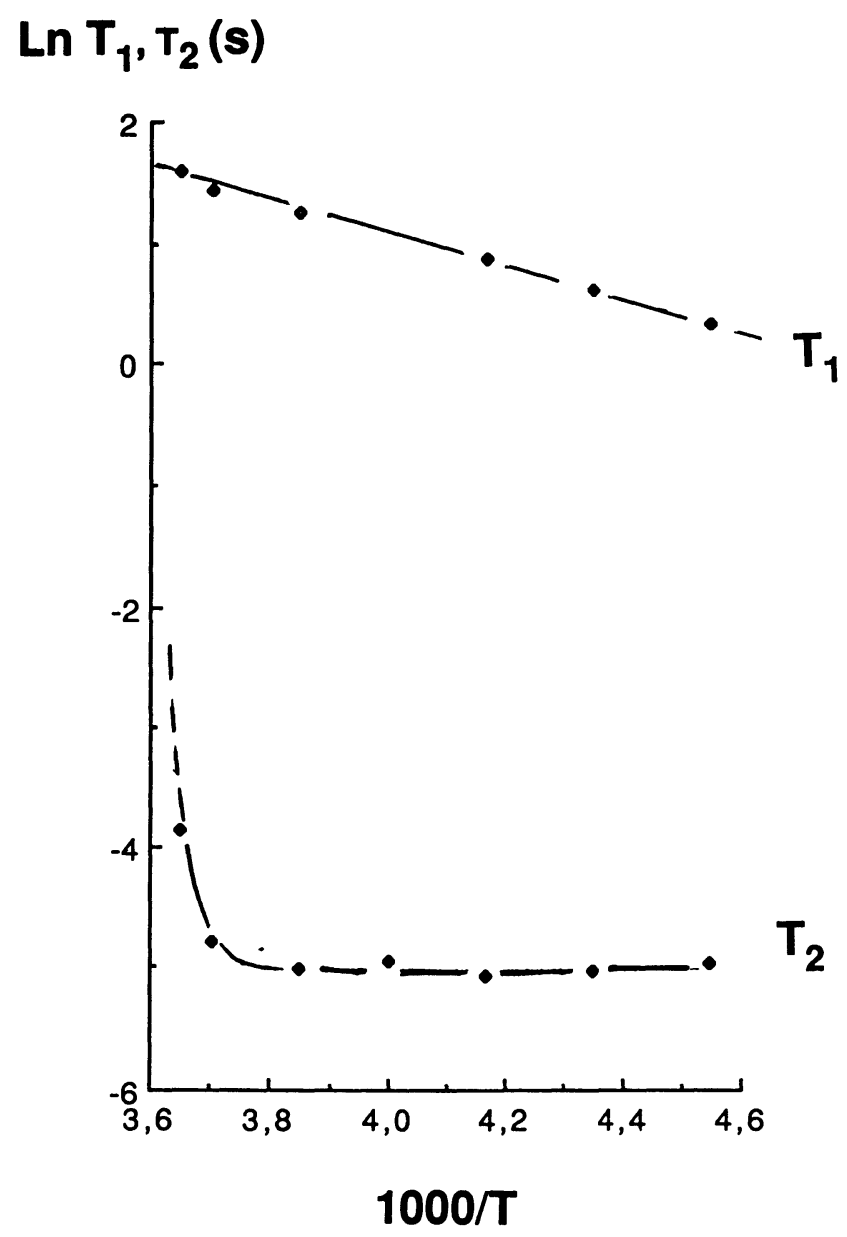

Fig. 7. - Spin-lattice $\left(T_{1}\right)$ and spin-spin $\left(T_{2}\right)$ relaxation times for the heptane line in the sample containing $1.5 \%$ of heptane in deuterated benzene.

inhomogeneity $\Delta H=3 \chi_{\mathrm{v}} H$ (where $\chi_{\mathrm{v}}$ is the magnetic susceptibility per unit volume), is to be expected in a sample consisting of loosely packed spheroids. This corresponds to a linewidth of roughly $1 \mathrm{ppm}$ in solid benzene, i.e. in our experiments $\sim 400 \mathrm{~Hz}$. If this extrabroadening is solely due to the internal field effect (we have checked, by using the shimming facility of our spectrometer, that the difference between $T_{2}$ and $T_{2}^{*}$ cannot be explained in terms of an inhomogeneity of the external magnetic field), than the linewidth is strictly proportional to the external field $H$. Using an identical Bruker spectrometer operating at $250 \mathrm{MHz}$ the narrow linewidth decreased by a factor of $\sim 1.25(500$ to $\sim 400 \mathrm{~Hz})$, instead of the expected 1.6 factor. It seems that the width of the narrow lines is not entirely dominated by the internal field effect. This is consistent with the $\left(T_{1}, T_{2}\right)$ data shown in figures 6 and 7 indicating that the local dipolar field due to the intermolecular interactions is not fully averaged to zero.

Above $T^{*}$ the progressive decrease of the linewidth of the narrow components and the progressive vanishing of the difference between $T_{2}$ and $T_{2}^{*}$ is consistent with the expected increasing mobility and the growing thickness of the quasi-liquid film.

Our conclusion that premelting is associated with extended defects like grain boundaries 


\section{$\%$ narrow line \% liquid}

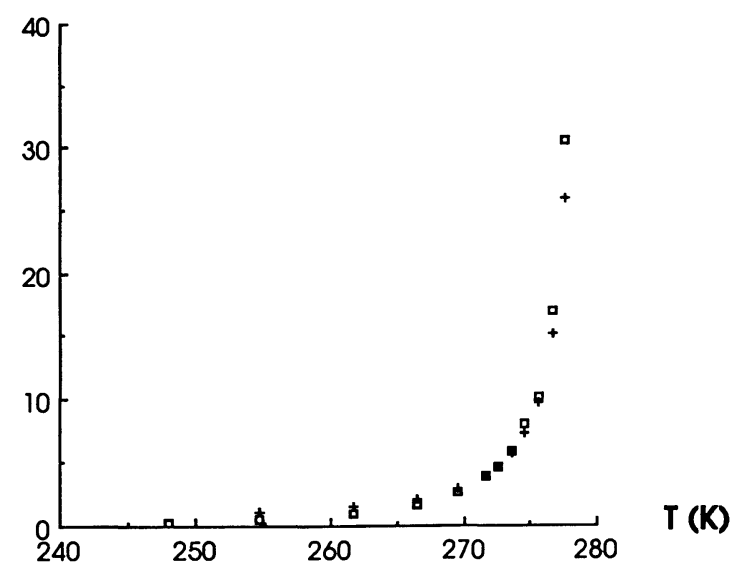

Fig. 8. - Proportion of the mobile molecules (benzene + heptane) involved in the proton n.m.r. narrow lines $(+)$ as a function of temperature together with the proportion of liquid $(\square)$ calculated from the phase diagram [6], in a sample of $x$ (heptane $)=0.002$.

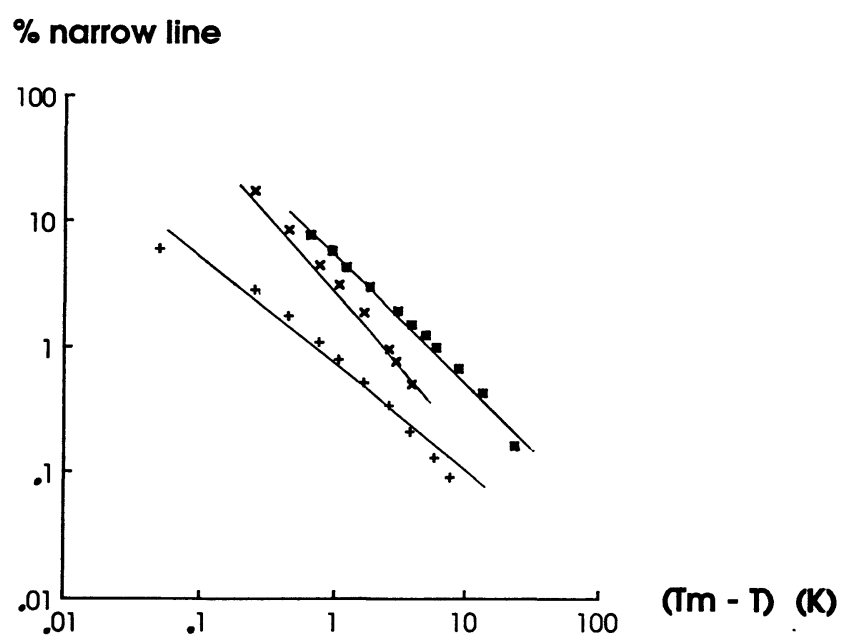

Fig. 9. - Proportion of mobile molecules as a function of temperature for 3 different purities : (+) $99.98 \%,(\times) 99.6 \%,(\square)$ benzene $+2 \%$ of heptane. The temperature dependence is well described by a power law : $\left(N_{\mathrm{m}} / N\right)=k\left(T_{\mathrm{m}}-T\right)^{-\gamma}$ where $k$ is a constant. The solid lines correspond to power law exponents of respectively : $\gamma=0.95$ (99.98\% sample), $\gamma=1.30$ (99.6\% sample), $\gamma=1.00$ (2\% heptane sample).

rather than point defects (vacancies), is supported by the n.m.r. self-diffusion work of Guillon and Conradi [13]. These authors have shown that, near melting, vacancy diffusion occurs at a rate of $\sim 10^{2} \mathrm{~Hz}$, while in polycristalline samples the pipe diffusion rate is about 4 orders of magnitude higher. The latter process occurs at a rate capable of affecting the width of the proton absorption line. Since, according to our model, the mobile molecules involved in the quasi-liquid stand at the grain boundaries, they are expected to diffuse at the pipe diffusion 
rate (or faster). This is consistent with the observed narrow lines appearing on top of the spectra.

In absence of heptane, the behaviour of the benzene samples (of purity 99.6 and $99.98 \%$ ) seems to be analogous to what is described above. As shown in figure 9, the proportion of mobile molecules $\left(N_{\mathrm{m}} / N\right)$ near melting is well described by a power law of the form $\left(N_{\mathrm{m}} / N\right)=k\left(T_{\mathrm{m}}-T\right)^{-\gamma}$, where $k$ is a constant. This had been noticed previously by Pruzan and coworkers [9]. Bartis [14] has shown that this equation can describe the growth of molten domains around crystal defects, considered from the point of view of the continuum theory of solids, with an exponent $\gamma$ between 1 and 2. In our preceeding work on thiophene [4] we found $\gamma=1.9$. In this work we found $\gamma$ values between 1 and 1.3. This difference is not well understood since we have no reason to believe, from our n.m.r. work, that the premelting process is different in benzene and thiophene. Here, however, the power law exponent, $\gamma$, is essentially identical in presence or absence of heptane, thus suggesting that our interpretation can be extended to the purest benzene samples. In this latter case the temperature $T^{*}$ for which the proportion of mobile molecules reaches $4 \%$ is very close to $T_{\mathrm{m}}$ (see Fig. 5) and this explains why the final narrowing of the thin line has not been observed.

\section{Conclusion.}

We suggest that, even with very pure material, premelting initiates at grain boundaries in solid benzene. This supports the general assumption that melting is an interface-initiated process and thus provides a convenient explanation for the systematic absence of observable superheating effects in solids.

In addition to our preceeding work on thiophene, we now have localized the molecular mobility domains, thanks to the study of heptane-benzene mixtures. Still the nature of the molten domains is not clear but is presumably analogous to what is obtained in the surface melting experiments, and sometimes called a "strongly correlated surface liquid ». More work is needed to clarify this point and we intend to investigate more closely the molecular mobilities by n.m.r. $\left({ }^{13} \mathrm{C}\right.$ and ${ }^{2} \mathrm{H}$ ) and molecular dynamics simulation.

Also the question of the influence of impurities is not fully resolved. A very small amount of solid-insoluble impurities seems to enhance the observed premelting phenomenon. Would this behaviour be the same in total absence of impurities is an open and rather difficult question since molecular solids are usually extremely hard to purify. Molecular dynamics simulation experiments should be of great help to clarify this point.

This model of impurity-assisted grain boundary premelting is presumably valid for thiophene [4], cyclohexanol [5] and p-dioxane [10]. We now intend to study the most " plastic » molecular solids like succinonitrile [11], for which the vacancy diffusion process seems to become prominant near melting.

\section{References}

[1] Frenken J. W. M. and Van Der Veen J. F., Phys. Rev. Lett. 54 (1985) 134 ; Pluis B., Frenken J. W. M. and Van Der Veen, Phys. Scr. 19B (1987) 382.

[2] BienfaIT M., Europhys. Lett. 4 (1987) 79.

[3] Bienfait M., Zeppenfeld P., Gay J. M. and Palmari J. P., Surf. Sci. 226 (1990) 327.

[4] Rousseau B., Bessada C. and Fuchs A. H., Solid State Commun. 67 (1988) 1017.

[5] Van Geet A. L., Anal. Chem. 42 (1970) 6.

[6] Clechet P. and Philippe R., Int. Data Series, Selected Data On Mixtures, Ser. A. 1980 (2) (1981) 116. 
[7] Andre D., Bessada C., Fuchs A. H., Rousseau B. and Szwarc H., J. Phys. France 49 (1988) 281.

[8] Fujara F., Petry W., Schnauss W. and Sillescu H., J. Chem. Phys. 89 (1988) 1801.

[9] Pruzan Ph., Liebenberg D. H. and Mills R. L., J. Phys. Chem. Solids 47 (1986) 949.

[10] Fried F., Molec. Cryst. Liquid. Cryst. 13 (1971) 279.

[11] Petrakis L. and RaO A., J. Chem. Phys. 39 (1964) 1633 ;

Dosseh G., Fressigne C., Rousseau B., Wilding N. and Fuchs A. H., to appear in J. Chim. Phys.

[12] Drain L. E., Proc. Phys. Soc. 80 (1962) 1380.

[13] Guillon T. and Conradi M. S., Phys. Rev. B 32 (1985) 7076.

[14] Bartis F. J., Philos. Mag. Lett. 60 (1989) 17. 